\title{
Cryptococcus neoformans: Tripping on Acid in the Phagolysosome
}

Carlos M. DeLeon-Rodriguez' ${ }^{1}$ and Arturo Casadevall ${ }^{1,2 *}$

1 Department of Microbiology and Immunology, Albert Einstein College of Medicine, Bronx, NY, USA, ${ }^{2}$ Department of

Molecular Microbiology and Immunology, Johns Hopkins University School of Public Health, Baltimore, MD, USA

Cryptococcus neoformans $(\mathrm{Cn})$ is a basidiomycetous pathogenic yeast that is a frequent cause of meningoencephalitis in immunocompromised individuals. $\mathrm{Cn}$ is a facultative intracellular pathogen in mammals, insects and amoeba. Cn infection occurs after inhalation of spores or desiccated cells from the environment. After inhalation Cn localizes to the lungs where it can be phagocytosed by alveolar macrophages. $\mathrm{Cn}$ is surrounded by a polysaccharide capsule that helps the fungus survive in vivo by interfering with phagocytosis, quenching free radical bursts and shedding polysaccharides that negatively modulates the immune system. After phagocytosis, Cn resides within the phagosome that matures to become a phagolysosome, a process that results in the acidification of the phagolysosomal lumen. Cn replicates at a higher rate inside macrophages than in the extracellular environment, possibly as a result that the phagosomal $\mathrm{pH}$ is near that optimal for growth. Cn increases the phagolysosomal $\mathrm{pH}$ and modulates the dynamics of Rab GTPases interaction with the phagolysosome. Chemical manipulation of the phagolysosomal $\mathrm{pH}$ with drugs can result in direct and indirect killing of $\mathrm{Cn}$ and reduced non-lytic exocytosis. Phagolysosomal membrane damage after $\mathrm{Cn}$ infection occurs both in vivo and in vitro, and is required for $\mathrm{Cn}$ growth and survival. Macrophage treatment with IFN- $\gamma$ reduces the phagolysosomal damage and increases intracellular killing of $\mathrm{Cn}$. Studies on mice and humans show that treatment with IFN- $\gamma$ can improve host control of the disease. However, the mechanism by which $\mathrm{Cn}$ mediates phagolysosomal membrane damage remains unknown but likely candidates are phospholipases and mechanical damage from an enlarging capsule. Here we review $\mathrm{Cn}$ intracellular interaction with a particular emphasis on phagosomal interactions and develop the notion that the extent of damage of the phagosomal membrane is a key determinant of the outcome of the Cn-macrophage interaction.

Keywords: Cryptococcus neoformans, macrophage, phagolysosomal membrane damage, pH, Interferon $\gamma$

\section{Cn AS A FACULTATIVE INTRACELLULAR PATHOGEN}

Cryptococcus neoformans ( $\mathrm{Cn}$ ), a basidiomycetous pathogenic yeast, is a relatively frequent cause of meningoencephalitis in immunocompromised individuals (Horgan et al., 1990; Thinyane et al., 2015). Cn is ubiquitous in the environment, inhabiting soils (Currie et al., 1994; Gugnani et al., 2005; Randhawa et al., 2008) and human infection occurs when aerosolized spores or desiccated fungal cells enter the lung via inhalation where $\mathrm{Cn}$ encounters the first line of defense: the alveolar macrophage (Feldmesser et al., 2000).Macrophages play a critical role in the pathogenesis of 
cryptococcosis, ranging from control of infection to possible roles in persistence, latency and extrapulmonary dissemination. Although historically $\mathrm{Cn}$ was divided into two varieties known as neoformans and gattii, genetic studies have subsequently separated these varieties into two species. The species Cryptococcus gattii has the potential to cause disease in immunocompetent individuals and animals (Stephen et al., 2002; Hoang et al., 2004). However, this review will only focus on $\mathrm{Cn}$, since most of the macrophage interaction studies have been done with $\mathrm{Cn}$. $\mathrm{Cn}$ is an facultative intracellular pathogen in such diverse hosts as mammals, amoebae (Steenbergen et al., 2001) and insects (Tenor et al., 2015; Trevijano-Contador et al., 2015), and employs various virulence factors to subvert cellular defense mechanisms. The manner in which $\mathrm{Cn}$ interacts with amoeba and macrophages is similar, suggesting that selection pressures in soil could lead to the emergence of particular traits that confer the capacity for virulence, thus making this microbe an accidental pathogen for mammals (Casadevall, 2012). In mammals, Cn was established to be a facultative intracellular pathogen in vivo and in vitro almost two decades ago (Feldmesser et al., 2001). In subsequent years, several groups have made major contributions to our understanding of the pathogenic strategy of $\mathrm{Cn}$ and those advances will be reviewed here.

The most distinctive feature of $\mathrm{Cn}$ is the expression of a large polysaccharide capsule that is a major virulence factor. The capsule functions in virulence through numerous mechanisms including preventing phagocytosis, quenching free radical bursts and interfering with immune responses (Bulmer and Sans, 1967; Zaragoza et al., 2008). Another mechanism by which $\mathrm{Cn}$ avoids phagocytosis is by the formation of titan cells, which prevent ingestion as a result of their enormous size (Okagaki et al., 2010; Zaragoza et al., 2010; Okagaki and Nielsen, 2012). The antiphagocytic function of the capsule is particularly relevant for intracellular pathogenesis since this process requires ingestion of the fungus by phagocytic cells. In the absence of opsonins, the capsule interferes with phagocytosis such that ingestion of encapsulated cells by macrophages is markedly lower (Macura et al., 2007). However, in the presence of capsule specific antibody and complement opsonins mediate efficient phagocytosis as described (Voelz and May, 2010). Although all encapsulated strains are opsonized by capsule binding antibodies, not all strains are efficiently opsonized by complement (Zaragoza et al., 2003). The mechanism for strain differences in complement opsonization involves differences in the geography of complement deposition in the capsule. If complement is deposited near the capsule surface, it is an effective opsonin, while complement deposition in the deeper layers of the capsule places complement component 3 in a location where it cannot interact with the complement receptor resulting in poor phagocytosis (Zaragoza et al., 2003). In addition, the capsule complement deposition pattern can be affected by the use of serum from different species, capsule size, and composition and the chronological age of the fungus (Young and Kozel, 1993; Gates and Kozel, 2006; Cordero et al., 2011). Therefore, the ability of $\mathrm{Cn}$ to increase its capsule size during infection is a mechanism that helps avoid complement-mediated phagocytosis.
$\mathrm{Cn}$ is able to survive and replicates at a higher rate inside macrophage than in the extracellular environment (Diamond and Bennett, 1973; Feldmesser et al., 2000). This ability of $\mathrm{Cn}$ to survive and replicate inside macrophages correlates with the virulence of clinical isolates, and is associated with dissemination via a Trojan horse hypothesis whereby Cn can cross the blood brain barrier inside macrophages (Charlier et al., 2009; Alanio et al., 2011). After infection, Cn can persist in the host in a latent state inside macrophages and multinucleated giant cells in granulomas. $\mathrm{Cn}$ in this latent state can emerge and cause disease if the host immune status change from immunocompetent to immunocompromised (Shibuya et al., 2005; Saha et al., 2007; Alanio et al., 2015). The macrophageCn interaction can have three major outcomes: (1) intracellular killing of Cn or control growth by the macrophage; (2) lysis of the macrophage and release of $\mathrm{Cn}$; and (3) non-lytic exocytosis in which both the macrophage and $\mathrm{Cn}$ survive (Figure 1). In addition, the phenomenon of macrophage to macrophage transfer of $\mathrm{Cn}$ cells has been described in vitro (Alvarez and Casadevall, 2007; Ma et al., 2007; Stukes et al., 2014). Of these possibilities exocytosis is the most common outcome (Stukes et al., 2014). Depletion of alveolar macrophage in rats and mice shows that the role of macrophages during $\mathrm{Cn}$ infection varies with the host species. Rat macrophages controlled $\mathrm{Cn}$ intracellular growth and were more resistant to pathogenmediated lysis. When rat lung macrophages were depleted the animals became more vulnerable (Shao et al., 2005). In contrast, murine macrophage served as a replicative niche for $\mathrm{Cn}$ and growth of $\mathrm{Cn}$ inside the macrophage can result in lysis of the macrophage (Shao et al., 2005). Differences in mouse strain susceptibility to $\mathrm{Cn}$ infection correlates with macrophage permissiveness for fungal intracellular replication (Zaragoza et al., 2007) but these differences are not well understood.

Acidification of the phagosome is an important macrophage antimicrobial mechanism but certain pathogens are able to adapt to overcome or exploit these acidic environments (Criscitiello et al., 2013). Pinpointing the mechanism by which $\mathrm{Cn}$ is able to survive within the harsh environment of the phagolysosome can provide insight to develop ways to reduce the intracellular replication of $\mathrm{Cn}$ and potentially prevent/treat disease. During infection, $\mathrm{Cn}$ experiences different $\mathrm{pH}$ levels and ability to survive in the host requires survival in the slightly alkaline environment of the blood and the cerebrospinal fluid, and the acidic environment of the phagolysosome. Consequently we will review the studies that explore the interactions of $\mathrm{Cn}$ in these diverse ranges of $\mathrm{pH}$ and phagosomal maturation.

\section{Cn LOCALIZATION INTO THE PHAGOLYSOSOME}

The localization of the microbe in tissue can play an important role on the virulence and survival of the pathogen. In tissues, such as lungs, that mount granulomatous responses, $\mathrm{Cn}$ is often found inside macrophages or in close approximation to them 


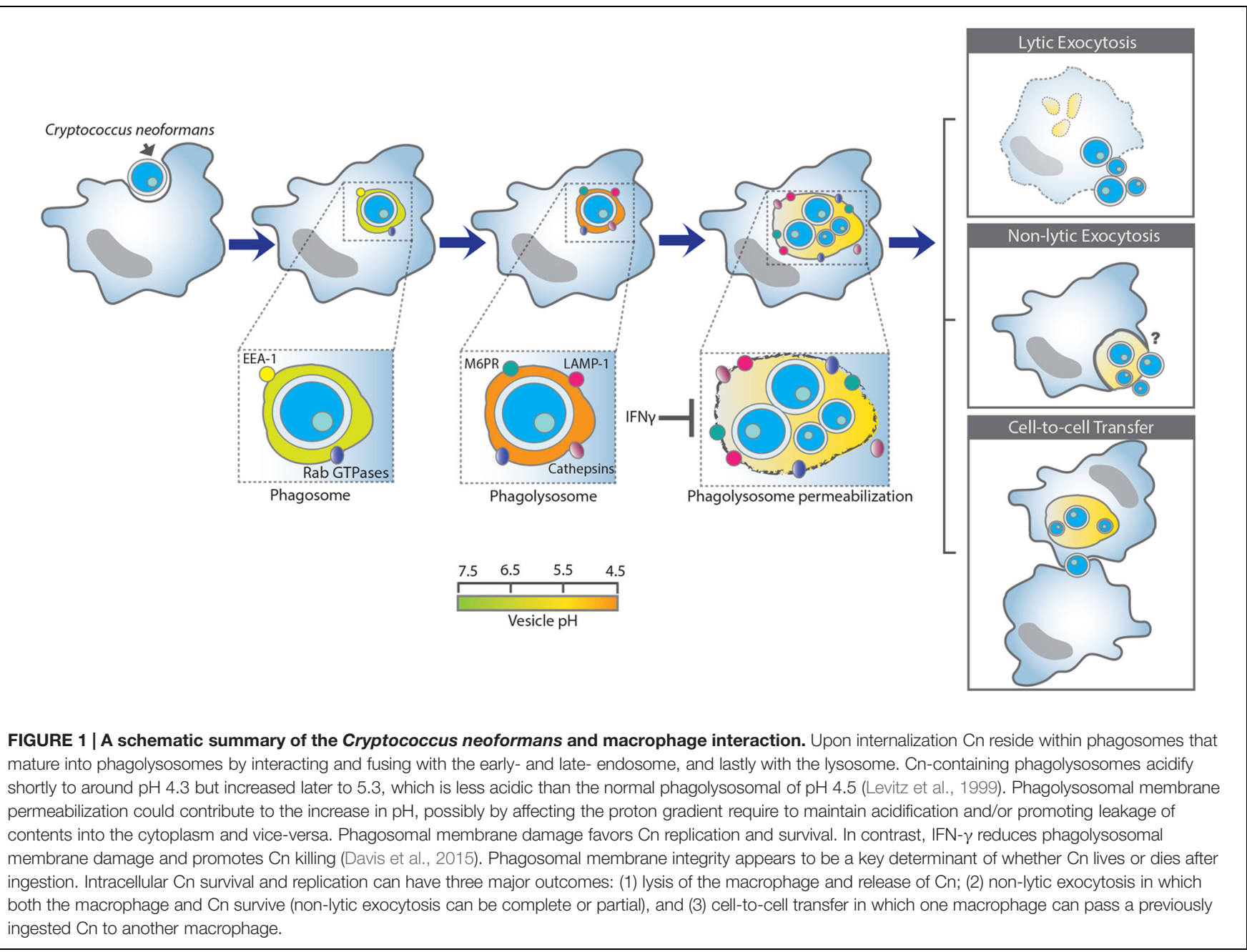

(Yamaoka et al., 1996; Kobayashi et al., 2001). To survive in their hosts, pathogenic microbes implement different strategies to avoid killing and degradation by the phagocytic cell, including inhibiting phagosome maturation (Clemens et al., 2000) or phagolysosome fusion (Horwitz, 1983), blocking phagosomal acidification (Horwitz and Maxfield, 1984) or escaping from the phagosome (Gaillard et al., 1987). By examining the localization of known endosomal and lysosomal markers, any inhibition of phagosome maturation or phagolysosome fusion during $\mathrm{Cn}$ infection can be observed. Studies done with $\mathrm{Cn}$-infected human monocyte-derived macrophage and J774.16 macrophage-like cells showed co-localization of the yeast with the lysosomalassociated membrane protein (LAMP-1), a lysosomal marker (Levitz et al., 1999; Alvarez and Casadevall, 2006). Cn-containing phagosomes in murine bone marrow derived dendritic cells and monocyte derived human dendritic cells fused with the early endosome marker (EEA-1) and LAMP-1 as early as 10 minutes after incubation with $\mathrm{Cn}$ (Wozniak and Levitz, 2008). In vivo studies also showed that phagolysosomal fusion occurred by two hours after infection in the alveolar macrophage in a murine model (Feldmesser et al., 2000). Furthermore, studies with bone marrow derived dendritic cells expressing CD63-mRFP1 and
Class II MHC-eGFP determined that these markers are recruited to $\mathrm{Cn}$-containing vacuoles. Thus, class II MHC proteins are recruited before CD63 and CD63 recruitment is dependent on the acidification of the phagosome (Artavanis-Tsakonas et al., 2006). In contrast, LAMP-1 recruitment is independent of phagosome acidification (Artavanis-Tsakonas et al., 2006). Cryptococcal phagolysosomes have some characteristics of autophagomes such as manifesting LC3 (microtubule-associated protein 1 light chain 3 alpha) co-localization (Nicola et al., 2012).

$\mathrm{Cn}$ intracellular trafficking is conserved in Drosophila melanogaster S2 cells and J774.A1 cells, upon internalization by both cell types $\mathrm{Cn}$ co-localized with EEA-1, the late endosome marker, mannose-6-phosphatase receptor (M6PR), LAMP-1 and Cathepsin D, another lysosomal marker (Qin et al., 2011). Furthermore, surfactant protein D was shown to enhance phagocytosis of acapsular cryptococcal mutants by macrophages in vitro but the resulting $\mathrm{Cn}$-containing phagolysosome shows a decrease in co-localization with LAMP-1 (Geunes-Boyer et al., 2009), suggesting that surfactant protein D influences Cncontaining phagosome maturation. Based on these studies, it was generally thought that $\mathrm{Cn}$ resided in the phagolysosome upon internalization by the macrophage and did not interfere 
with phagosome maturation. However, new evidence based on the analysis of the Rab GTPases localization, which are early endosomes and phagosomes markers, revealed a different pattern for the interaction of these molecules with phagosomes containing live $\mathrm{Cn}$, heat killed $\mathrm{Cn}$ or latex beads, suggesting that $\mathrm{Cn}$ influenced phagosome maturation. Rab 5 and 11 are recruited to the Cn-containing vesicle shortly after phagocytosis but their presence diminishes more rapidly in phagosomes containing live fungal cells than in those containing latex beads- or heat killed Cn-cells (Smith et al., 2015). However, the extent to which Cn affects phagosome maturation is unknown but it is likely that the effect is smaller than other pathogens, like Mycobacteria tuberculosis, which directly interfere with phagosome maturation by blocking fusion with late endosomes and lysosomes (Kelley and Schorey, 2003).

Phagosomal maturation and acidification are required for both the localization and optimal enzymatic activity of lysosomal proteases, respectively (Boya and Kroemer, 2008). Cn localization in the phagolysosome exposes the yeast to cathepsins, other hydrolases, and reactive oxygen species in the lysosomal lumens. Cathepsins are lysosome related proteases with a role in lysosomal protein recycling, Toll-like receptors signaling, extracellular matrix degradation, activation and inhibition of cytokines, antigen processing, and more recently have been implied in apoptosis. To activate apoptosis, cathepsins need to be released from the lysosomes into the cytosol via lysosomal membrane permeabilization (Conus and Simon, 2010). Lysosomal extracts purified from bone marrow derived dendritic cells can kill $\mathrm{Cn}$ in a dose dependent manner (Wozniak and Levitz, 2008). Analysis of purified lysosomal enzymes showed that Cathepsin B and Cathepsin L inhibited $\mathrm{Cn}$ growth, and that an inhibitor of Cathepsin B enzymatic activity surprisingly increased inhibition of $\mathrm{Cn}$ growth (Hole et al., 2012). These results suggested that $\mathrm{Cn}$ exposure to lysosomal proteases, specifically cathepsin B and L inhibited cryptococcal growth. In vivo analysis of Cathepsins $\mathrm{B}$ and Cathepsin L activity in Cn-containing phagolysosome has shown little evidence of enzymatic activity, but macrophages challenged with heat killed $\mathrm{Cn}$ show Cathepsin L activity (Smith et al., 2015). The discrepancy between these results might be attributed to the fact that in the first studies Cn was incubated in vitro with lysosomal extracts whereas in the later studies the analysis was done in infected cells. The Cn capsule confers resistance against reactive oxygen species (Zaragoza et al., 2008) and presumably protects the fungal cell during the lysosomal oxidative burst. However, as to whether the capsule also provides resistance against cathepsins and others proteases remain unclear. In this regard, the dense polysaccharide fibrillar network that surrounds the cell could provide protection by trapping cytotoxic enzymes but this mechanism remains to be shown.

In summary, the difference between cathepsin activity in phagosomes containing live and dead $\mathrm{Cn}$ suggests that $\mathrm{Cn}$ can affect cathepsin enzymatic activity but the mechanism is unknown. Given that phagosomes containing live $\mathrm{Cn}$ become progressively leaky during intracellular residence, one possible explanation for the discordance between the results obtained with live and dead $\mathrm{Cn}$ is that the negative cathepsin activity observed with the live cells is lost with a degraded phagosomal membrane.

\section{EFFECT OF pH ON THE Cn GROWTH (STUDIES INDEPENDENT OF HOST)}

The response of $\mathrm{Cn}$ to $\mathrm{pH}$ is important in pathogenesis because the fungus goes from neutral to slightly alkaline conditions in extracellular body fluids to acidic conditions in the mature phagolysosomal compartment. An inverse correlation between growth of $\mathrm{Cn}$ and the $\mathrm{pH}$ of the growth medium was initially established more than 60 years ago (Mosberg and Mc, 1951; Howard, 1961), when several investigators reported that acidic milieus enhanced growth while alkaline conditions inhibited growth. More recent studies have confirmed those results by showing that $\mathrm{Cn}$ can grow in the $\mathrm{pH}$ range of 5-8 and also revealed that the optimal growth for $\mathrm{Cn}$ is at pH 5 (Levitz et al., 1997). These studies also established differences in the susceptibility of $\mathrm{Cn}$ strains to $\mathrm{pH}$ and a dependence on temperature such that fungal cells were less resistant at $37^{\circ}$ to alkaline $\mathrm{pH}$. However, a $\mathrm{Cn}$ strain deficient in the $\mathrm{Ca}^{+2}$-regulated protein Calcineurin is more susceptible to alkaline $\mathrm{pH}$, and become avirulent in a rabbit model of cryptococcal meningitis (Odom et al., 1997). Interestingly, a Cn strain lacking glycosphingolipid glucosylceramide was avirulent in a murine animal model when challenged intranasally but was virulent when the infection was done intravenously, suggesting that the lung environment controlled the infection. The glycosphingolipid glucosylceramide mutant was arrested in the $\mathrm{S}$ and $\mathrm{G}_{2} / \mathrm{M}$ phase at $\mathrm{pH} 7.4$ in $5 \% \mathrm{CO}_{2}$, but is not affected at $\mathrm{pH} 4$ in $5 \% \mathrm{CO}_{2}$ (Rittershaus et al., 2006). One explanation for these observations is that during the intranasal infection the glycosphingolipid glucosylceramide mutant is not able to replicate in the alkaline extracellular environment of the lung. Those fungal cells that are ingested by the alveolar macrophage can replicate in the acidic environment of the phagolysosome but these are controlled by the formation of granulomas. In contrast, after intravenous infection the glycosphingolipid glucosylceramide mutant growth was also arrested in the alkaline environment of the blood, but was able to replicate once it invaded other organs and reside in abscess with an acidic environment optimal for the growth of the mutant (Rittershaus et al., 2006). These studies suggest that the ability of $\mathrm{Cn}$ to grow at different $\mathrm{pH}$ is important for pathogenicity.

The conserved Rim101 pathway mediates fungal response to extracellular neutral/alkaline $\mathrm{pH}$, for reviews see (Davis, 2009; Cornet and Gaillardin, 2014). Extensive work has been done to identify the homologues of the Rim101 pathway and the role of Rim101 pathway during Cn-host interaction. Cn Rim101 mutants show a capsule defect, altered cell wall composition and increased susceptibility to different host induced stress stimulus (O'Meara et al., 2014). Recently, the Rim pathway was shown to be activated by the increase of $\mathrm{pH}$, with the protein $\mathrm{Rral}$ functioning as a $\mathrm{pH}$ sensor, but others components of the $\mathrm{pH}$ sensing complex have not been identified (Ost et al., 2015). 
Further studies of this pathway may shed light unto how $\mathrm{Cn}$ is able to modulate gene expression to survive between the different $\mathrm{pH}$ environments it encounter during host infection.

\section{Cn AND PHAGOLYSOSOMAL ACIDIFICATION}

Phagosome maturation results in the acidification of the phagosomal lumen, creating an optimal environment for the proteases recruited to that site during the maturation process. In the next two sections, we review what is known about phagosomal acidification during $\mathrm{Cn}$ infection of phagocytic cells and the effect of chemical modulation of phagosomal acidification on the outcome of $\mathrm{Cn}$ infection. Initial studies measuring in vivo phagolysosomal $\mathrm{pH}$ done in rabbit alveolar macrophages after $24 \mathrm{~h}$ infection using fluorescein-labeled heat-killed cryptococcal cells revealed an average $\mathrm{pH} \quad 5$ and 5.2 for phagolysosomes containing heat killed $\mathrm{Cn}$ and fluorescein-labeled silica particles, respectively. They noted that approximately $2 \%$ of the phagolysosomes containing heat-killed $\mathrm{Cn}$ had a $\mathrm{pH}$ of 6.5 , which never occurred with the silica particles (Nessa et al., 1997). The $\mathrm{pH}$ of $\mathrm{Cn}$-containing phagolysosomes was also measured in monocyte-derived macrophage using live $\mathrm{Cn}$ and heat-killed $\mathrm{Cn}$ after 3 and $24 \mathrm{~h}$ infection. The $\mathrm{pH}$ of phagolysosomes containing heat-killed $\mathrm{Cn}$ remained stable over time, ranging from 5.2 to 5.1 , but the $\mathrm{pH}$ of phagolysosomes containing live $\mathrm{Cn}$ increased from 4.3 to 5.3 , which closely matches the optimal $\mathrm{pH}$ for fungal growth. The phagolysosomal $\mathrm{pH}$ of neutrophils infected with live $\mathrm{Cn}$ remained constant over a $3 \mathrm{~h}$ period hovering between 5.2 and 5.0 (Levitz et al., 1999). A more recent study suggests that live Cn, but not heat-killed $\mathrm{Cn}$, could block acidification of the phagolysosome (Smith et al., 2015). The differences in these studies could reflect the use of different cell models, Cn strains, and/or technical approaches. Cn mutants in phospholipase B, Sec14 secretion system, urease expression, and the acapsular mutant maintained their ability to prevent acidification, suggesting that prevention of acidification occurred through an independent process that is unrelated to those virulence factors (Smith et al., 2015). However, a strain of $\mathrm{Cn}$ that overexpressed the antifungal resistance protein 1 (AFR1) delayed the acidification of the phagolysosome and resided in phagolysosomes with a lower degree of co-localization with Rab5-, Rab7- and LAMP2 (Orsi et al., 2009). In summary, $\mathrm{Cn}$ growth is affected by $\mathrm{pH}$ but the acidity of the phagolysosomal compartment does not appear to be a significant mechanism for microbial control and may in fact promote fungal replication.

\section{Cn AND CHEMICAL MANIPULATION OF PHAGOSOMAL ACIDIFICATION}

Experiments done in the late 1990s showed that treatment of BV2 microglial cells with the weak bases, chloroquine, and ammonium chloride, enhanced the anticryptococcal activity of microglial cells. These weak bases act as lysosomotropic agents by accumulating in the lysosomal compartment (Villamil Giraldo et al., 2014). Similar effects were observed when microglial cells were treated with bafilomycin A1, an inhibitor of the vacuolartype $\mathrm{H}^{+}$-ATPases. These investigators also demonstrated an increase in the median survival time of mice treated with an intracerebral administration of chloroquine before challenge with a lethal dose of Cn (Mazzolla et al., 1997). Similar results were noted when treating human monocyte-derived macrophages (MDM) with chloroquine and ammonium chloride, which increased anticryptoccocal activity of the MDM independent of iron deprivation. Chloroquine enhancement of anticryptococcal activity was also observed with monocytes derived from HIVseronegative and HIV-seropositive donors, and in a murine model of experimental cryptococcosis using immunocompetent and immunodeficient mice (Levitz et al., 1997). Chloroquine treatment increased the $\mathrm{pH}$ of phagosomes containing heatkilled $\mathrm{Cn}$ in a dose dependent manner showing $\mathrm{pH}$ values of approximately 5.2 at $1 \mu \mathrm{M}, 6.5$ at $10 \mu \mathrm{M}$, and 7.5 at $100 \mu \mathrm{M}$ (Levitz et al., 1999). Interestingly, both chloroquine and quinacrine accumulate within $\mathrm{Cn}$ and directly inhibit its growth. Accumulation of chloroquine and quinacrine within $\mathrm{Cn}$ increased at physiological conditions, but the mechanism by which it exerts its anticryptococcal activity remains unknown (Harrison et al., 2000). Cn growth was also inhibited by ammonium chloride and bafilomycin $\mathrm{A}$ in a concentration dependent manner (Harrison et al., 2000). These results suggest that treatment of phagocytic cells with lysosomotropic agents had a direct effect on $\mathrm{Cn}$ as well as an alternative indirect effect by increasing the $\mathrm{pH}$ of the $\mathrm{Cn}$-containing phagolysosome (Harrison et al., 2000; Weber et al., 2000).

Non-lytic exocytosis can occur after phagocytosis of $\mathrm{Cn}$ by macrophages, and results on the expulsion of viable $\mathrm{Cn}$ to the extracellular environment without the lysis of the macrophage. Blockage of phagosome maturation using Concanamycin A, an inhibitor of V-ATPase, reduced $\mathrm{Cn}$ non-lytic exocytosis (Ma et al., 2006). Macrophage treatment with bafilomycin A revealed a slight decrease in non-lytic exocytosis, while treatment with ammonium chloride and chloroquine significantly increase nonlytic exocytosis (Ma et al., 2006; Nicola et al., 2011; Qin et al., 2011). The mechanism for how $\mathrm{pH}$ affects non-lytic exocytosis remains unexplained.

\section{Cn AND PHAGOLYSOSOME MEMBRANE PERMEABILIZATION OR DAMAGE}

As a consequence of lysosomal membrane permeabilization, cathepsins and other proteases are released from the lysosomal lumen into the cytosol where they can activate cell death (Boya and Kroemer, 2008). Phagolysosomal membrane damage was observed in alveolar macrophage of mice infected with $\mathrm{Cn}$ at 7 days post-infection (Feldmesser et al., 2000). Subsequent studies revealed that $\mathrm{Cn}$-containing phagosomes pre-loaded with fluorescently labeled dextran showed diffusion of the fluorescent signal, indicative of leakage of phagosomal contents into the cytoplasm. Lysosomal membrane permeabilization was confirmed by demonstrating the inability of the Cn-containing 
phagosome to maintain an acidic environment (Tucker and Casadevall, 2002). The mechanisms responsible for phagolysosomal permeability are unknown. Cn extracellular phospholipase activity was hypothesized to have a role on the degradation of the phagolysosomal membrane but this effect was not experimentally demonstrated (Cox et al., 2001). Nonetheless, $\mathrm{Cn}$ phagolysosomal damage is associated with cryptococcal replication and survival, but activation of macrophages with IFN- $\gamma$ can reduce phagolysosomal damage (Davis et al., 2015). In Cn-infected THP-1 macrophage-like cells, phagolysosomal membrane permeabilization induces formation of the adaptor protein apoptosis-associated speck-like protein containing a CARD speck, suggesting that release of phagolysosomal content, including Cathepsin B, activates inflammasomes resulting in processing and release of IL-1 $\beta$. Treatment with Cathepsin B inhibitor reduced IL-1 $\beta$ secretion implying that phagolysosomal damage is required for activation of the canonical caspase- 8 inflammasome (Chen et al., 2015). Taken together, internalized $\mathrm{Cn}$ induces phagolysosomal membrane permeabilization and leads to host cell death in a manner dependent on inflammasomes activation.

\section{CONCLUSION AND PERSPECTIVE}

During the preparation of this review, it was apparent that the literature is inconsistent with how it refers to the phagolysosome and the timing of its appearance. According to LAMP-1 staining, the $\mathrm{Cn}$-containing phagosome fuses with the lysosome as early as one hour post incubation, implying that $\mathrm{Cn}$ resides inside the phagolysosome by one-hour post ingestion. To avoid confusion during this review, we used the term phagolysosome whenever we referred to studies that used experimental time of onehour post incubation or longer. Readers should note that phagosomal maturation is a dynamic process that may vary between individual phagosomes. For example, EEA1 and LAMP1 were each associated with some phagosomes at early time of macrophage infection but the number of phagosomes positive for these markers increased gradually with time (Wozniak and Levitz, 2008). Phagosomal acidification is a critical step during phagosomal maturation to allow phagosome-lysosome fusion and provide an optimal environment for the activity of antimicrobial enzymes. However, internalization of $\mathrm{Cn}$ results in a decrease of phagolysosomal $\mathrm{pH}$ shortly after ingestion, which is followed by an increase in phagolysosomal $\mathrm{pH}$ over time culminating in an inability of the $\mathrm{Cn}$-containing phagolysosome to maintain the acidic $\mathrm{pH}$ as a result of membrane damage (Levitz et al., 1999; Tucker and Casadevall, 2002). Further studies are needed to determine the precise relationship between changes in $\mathrm{pH}$ in the $\mathrm{Cn}$-containing phagolysosome, $\mathrm{Cn}$ growth and the onset of phagolysosomal membrane damage. Future studies should take in consideration the effect of phagolysosomal membrane permeabilization with regards to the acidification of the Cn-containing phagolysosome and determined causal and temporal relationships, if any. If phagolysosomal membrane permeabilization occurs only in a fraction of those Cn-containing phagolysosome, it is possible that will result in a gradient of $\mathrm{pH}$ values, as damage of the phagolysosomal membrane will result in neutralization of phagolysosome acidification by cytoplasmic contents.

Phagolysosome membrane damage can promote Cn growth after cell ingestion (Davis et al., 2015), presumably by disabling microbicide mechanisms or damaging the host cells through the spillage of vesicular content into the cytoplasm. The mechanism for $\mathrm{Cn}$ induction of phagolysosome membrane damage remains unknown but various hypotheses have been discussed in the literature. These include the notion that phagolysosomal membrane damage is a result of $\mathrm{Cn}$ replication and capsular growth that produce physical damage (Feldmesser et al., 2000). Secondly, secreted fungal proteins damage the phagolysosomal membrane directly. In this regard, extracellular phospholipase was suggested as a candidate for phagolysosomal membrane damage (Cox et al., 2001). Phospholipids induce enlargement of $\mathrm{Cn}$ capsule which also requires phospholipase $\mathrm{B}$ activity (Chrisman et al., 2011). It is possible that there is a synergistic effect that combines damage of the phagolysosomal membrane by the extracellular fungal phospholipase activity and physical damage of the phagolysosomal membrane by growth of the fungal capsule induced by the phospholipase products.

$\mathrm{Cn}$ intracellular residence was shown to result in damage to a variety of cellular systems including mitochondrial function (Coelho et al., 2015). The amount of damage incurred by the host cell may depend on the degree of cellular activation. In this regard, treatment of macrophage with IFN- $\gamma$ was shown to protect the phagolysosomal membrane from damage and promoted the anti-fungicidal ability of the macrophages (Davis et al., 2015). Previous studies shows that IFN- $\gamma$ also increased anti-fungicidal activity in rat alveolar macrophage and natural killer cells, and increase survival time in two murine models of Cn infection (Mody et al., 1991; Salkowski and Balish, 1991; Kawakami et al., 1995, 1996). IFN- $\gamma$ administrations were shown to prolong mice survival when used as an adjuvant treatment in combination with amphotericin B in normal mice and SCID mice infected with Cn (Joly et al., 1994; Lutz et al., 2000; Clemons et al., 2001). IFN- $\gamma$ has been used clinically as adjuvant treatment and was shown to improve in $\mathrm{Cn}$ clearance from the cerebrospinal fluid in HIV-positive patients with cryptococcal meningitis (Jarvis et al., 2012). These observations in rodents and humans suggest that interventions that promote phagosomal membrane integrity could have potential therapeutic applications.

In summary, it appears that the phagolysosomal membrane is a key battleground in the struggle between $\mathrm{Cn}$ and phagocytic cells. Damage to the membrane with loss of acidity and spillage of phagolysosomal contents into the cytoplasm favors the fungus and catalyzes as series of events that compromise the host cells and interfere with their ability to control infection. On the other hand, integrity of the phagolysosomal membrane is associated with control of infection. At this time the factors that tip the balance toward membrane damage or integrity are poorly understood and their elucidation is a research priority in the field. The understanding of this process is given additional urgency since therapeutic interventions to stabilize the phagolysosome may tip the balance to the benefit of the host. 


\section{AUTHOR CONTRIBUTIONS}

CD-R and AC collaborated to write the manuscript.

\section{FUNDING}

AC is supported by 5R01HL059842, 5R01AI033774, 5R37AI033142, and 5R01AI052733.

\section{REFERENCES}

Alanio, A., Desnos-Ollivier, M., and Dromer, F. (2011). Dynamics of Cryptococcus neoformans-macrophage interactions reveal that fungal background influences outcome during cryptococcal meningoencephalitis in humans. MBio 2:e00158-11. doi: 10.1128/mBio.00158-11

Alanio, A., Vernel-Pauillac, F., Sturny-Leclere, A., and Dromer, F. (2015). Cryptococcus neoformans host adaptation: toward biological evidence of dormancy. MBio 6:e02580-14. doi: 10.1128/mBio.02580-14

Alvarez, M., and Casadevall, A. (2006). Phagosome extrusion and host-cell survival after Cryptococcus neoformans phagocytosis by macrophages. Curr. Biol. 16, 2161-2165. doi: 10.1016/j.cub.2006.09.061

Alvarez, M., and Casadevall, A. (2007). Cell-to-cell spread and massive vacuole formation after Cryptococcus neoformans infection of murine macrophages. BMC Immunol. 8:16. doi: 10.1186/1471-2172-8-16

Artavanis-Tsakonas, K., Love, J. C., Ploegh, H. L., and Vyas, J. M. (2006). Recruitment of CD63 to Cryptococcus neoformans phagosomes requires acidification. Proc. Natl. Acad. Sci. U.S.A. 103, 15945-15950. doi: 10.1073/pnas.0607528103

Boya, P., and Kroemer, G. (2008). Lysosomal membrane permeabilization in cell death. Oncogene 27, 6434-6451. doi: 10.1038/onc.2008.310

Bulmer, G. S., and Sans, M. D. (1967). Cryptococcus neoformans. II. Phagocytosis by human leukocytes. J. Bacteriol. 94, 1480-1483.

Casadevall, A. (2012). Amoeba provide insight into the origin of virulence in pathogenic fungi. Adv. Exp. Med. Biol. 710, 1-10. doi: 10.1007/978-1-44195638-5_1

Charlier, C., Nielsen, K., Daou, S., Brigitte, M., Chretien, F., and Dromer, F. (2009). Evidence of a role for monocytes in dissemination and brain invasion by Cryptococcus neoformans. Infect. Immun. 77, 120-127. doi: 10.1128/IAI. 01065-08

Chen, M., Xing, Y., Lu, A., Fang, W., Sun, B., Chen, C., et al. (2015). Internalized Cryptococcus neoformans activates the canonical caspase-1 and the noncanonical caspase-8 inflammasomes. J. Immunol. 195, 4962-4972. doi: 10.4049/jimmunol.1500865

Chrisman, C. J., Albuquerque, P., Guimaraes, A. J., Nieves, E., and Casadevall, A. (2011). Phospholipids trigger Cryptococcus neoformans capsular enlargement during interactions with amoebae and macrophages. PLoS Pathog. 7:e1002047. doi: 10.1371/journal.ppat.1002047

Clemens, D. L., Lee, B. Y., and Horwitz, M. A. (2000). Mycobacterium tuberculosis and Legionella pneumophila phagosomes exhibit arrested maturation despite acquisition of Rab7. Infect. Immun. 68, 5154-5166. doi: 10.1128/IAI.68.9.51545166.2000

Clemons, K. V., Lutz, J. E., and Stevens, D. A. (2001). Efficacy of recombinant gamma interferon for treatment of systemic cryptococcosis in SCID mice. Antimicrob. Agents Chemother. 45, 686-689. doi: 10.1128/AAC.45.3.686689.2001

Coelho, C., Souza, A. C., Derengowski Lda, S., de Leon-Rodriguez, C., Wang, B., Leon-Rivera, R., et al. (2015). Macrophage mitochondrial and stress response to ingestion of Cryptococcus neoformans. J. Immunol. 194, 2345-2357. doi: 10.4049/jimmunol.1402350

Conus, S., and Simon, H. U. (2010). Cathepsins and their involvement in immune responses. Swiss. Med. Wkly 140, w13042. doi: 10.4414/smw.2010. 13042

Cordero, R. J., Pontes, B., Guimaraes, A. J., Martinez, L. R., Rivera, J., Fries, B. C., et al. (2011). Chronological aging is associated with biophysical and

\section{ACKNOWLEDGMENTS}

We will like to express our gratitude to Dr. Yesseinia Angleró-Rodríguez for the preparation of Figure $\mathbf{1}$ and to Dr. Man Shun Fu, Dr. Carolina Coelho, and Dr. Johanna Rivera for their insightful comments and critique during the preparation of this manuscript. The title of this manuscript was inspired by the title of another paper (Criscitiello et al., 2013).

chemical changes in the capsule of Cryptococcus neoformans. Infect. Immun. 79, 4990-5000. doi: 10.1128/IAI.05789-11

Cornet, M., and Gaillardin, C. (2014). pH signaling in human fungal pathogens: a new target for antifungal strategies. Eukaryot. Cell 13, 342-352. doi: 10.1128/EC.00313-13

Cox, G. M., McDade, H. C., Chen, S. C., Tucker, S. C., Gottfredsson, M., Wright, L. C., et al. (2001). Extracellular phospholipase activity is a virulence factor for Cryptococcus neoformans. Mol. Microbiol. 39, 166-175. doi: 10.1046/j.13652958.2001.02236.x

Criscitiello, M. F., Dickman, M. B., Samuel, J. E., and de Figueiredo, P. (2013). Tripping on acid: trans-kingdom perspectives on biological acids in immunity and pathogenesis. PLoS Pathog. 9:e1003402. doi: 10.1371/journal.ppat.10 03402

Currie, B. P., Freundlich, L. F., and Casadevall, A. (1994). Restriction fragment length polymorphism analysis of Cryptococcus neoformans isolates from environmental (pigeon excreta) and clinical sources in New York City. J. Clin. Microbiol. 32, 1188-1192.

Davis, D. A. (2009). How human pathogenic fungi sense and adapt to $\mathrm{pH}$ : the link to virulence. Curr. Opin. Microbiol. 12, 365-370. doi: 10.1016/j.mib.2009.05.006

Davis, M. J., Eastman, A. J., Qiu, Y., Gregorka, B., Kozel, T. R., Osterholzer, J. J., et al. (2015). Cryptococcus neoformans-induced macrophage lysosome damage crucially contributes to fungal virulence. J. Immunol. 194, 2219-2231. doi: 10.4049/jimmunol.1402376

Diamond, R. D., and Bennett, J. E. (1973). Growth of Cryptococcus neoformans within human macrophages in vitro. Infect. Immun. 7, 231-236.

Feldmesser, M., Kress, Y., Novikoff, P., and Casadevall, A. (2000). Cryptococcus neoformans is a facultative intracellular pathogen in murine pulmonary infection. Infect. Immun. 68, 4225-4237. doi: 10.1128/IAI.68.7.4225-42 37.2000

Feldmesser, M., Tucker, S., and Casadevall, A. (2001). Intracellular parasitism of macrophages by Cryptococcus neoformans. Trends Microbiol. 9, 273-278. doi: 10.1016/S0966-842X(01)02035-2

Gaillard, J. L., Berche, P., Mounier, J., Richard, S., and Sansonetti, P. (1987). In vitro model of penetration and intracellular growth of Listeria monocytogenes in the human enterocyte-like cell line Caco-2. Infect. Immun. 55, 2822-2829.

Gates, M. A., and Kozel, T. R. (2006). Differential localization of complement component 3 within the capsular matrix of Cryptococcus neoformans. Infect. Immun. 74, 3096-3106. doi: 10.1128/IAI.01213-05

Geunes-Boyer, S., Oliver, T. N., Janbon, G., Lodge, J. K., Heitman, J., Perfect, J. R., et al. (2009). Surfactant protein D increases phagocytosis of hypocapsular Cryptococcus neoformans by murine macrophages and enhances fungal survival. Infect. Immun. 77, 2783-2794. doi: 10.1128/IAI.00 088-09

Gugnani, H. C., Mitchell, T. G., Litvintseva, A. P., Lengeler, K. B., Heitman, J., Kumar, A., et al. (2005). Isolation of Cryptococcus gattii and Cryptococcus neoformans var. grubii from the flowers and bark of Eucalyptus trees in India. Med. Mycol. 43, 565-569. doi: 10.1080/13693780500160785

Harrison, T. S., Griffin, G. E., and Levitz, S. M. (2000). Conditional lethality of the diprotic weak bases chloroquine and quinacrine against Cryptococcus neoformans. J. Infect. Dis. 182, 283-289. doi: 10.1086/315649

Hoang, L. M., Maguire, J. A., Doyle, P., Fyfe, M., and Roscoe, D. L. (2004) Cryptococcus neoformans infections at vancouver hospital and health sciences centre (1997-2002): epidemiology, microbiology and histopathology. J. Med. Microbiol. 53, 935-940. doi: 10.1099/jmm.0.05427-0 
Hole, C. R., Bui, H., Wormley, F. L. Jr., and Wozniak, K. L. (2012). Mechanisms of dendritic cell lysosomal killing of Cryptococcus. Sci. Rep. 2:739. doi: 10.1038/srep00739

Horgan, M., Boothman, S., Keane, C. T., Temperley, I. J., Mulcahy, F., and Jackson, F. (1990). Cryptococcal meningitis occurring in HIV infected individuals. Ir. Med. J. 83, 157-158.

Horwitz, M. A. (1983). The Legionnaires' disease bacterium (Legionella pneumophila) inhibits phagosome-lysosome fusion in human monocytes. J. Exp. Med. 158, 2108-2126. doi: 10.1084/jem.158.4.1319

Horwitz, M. A., and Maxfield, F. R. (1984). Legionella pneumophila inhibits acidification of its phagosome in human monocytes. J. Cell Biol. 99, 1936-1943. doi: $10.1083 /$ jcb.99.6.1936

Howard, D. H. (1961). Some factors which affect the initiation of growth of Cryptococcus neoformans. J. Bacteriol. 82, 430-435.

Jarvis, J. N., Meintjes, G., Rebe, K., Williams, G. N., Bicanic, T., Williams, A., et al. (2012). Adjunctive interferon-gamma immunotherapy for the treatment of HIV-associated cryptococcal meningitis: a randomized controlled trial. AIDS 26, 1105-1113. doi: 10.1097/QAD.0b013e3283536a93

Joly, V., Saint-Julien, L., Carbon, C., and Yeni, P. (1994). In vivo activity of interferon-gamma in combination with amphotericin B in the treatment of experimental cryptococcosis. J. Infect. Dis. 170, 1331-1334. doi: 10.1093/infdis/170.5.1331

Kawakami, K., Kohno, S., Kadota, J., Tohyama, M., Teruya, K., Kudeken, N., et al. (1995). T cell-dependent activation of macrophages and enhancement of their phagocytic activity in the lungs of mice inoculated with heat-killed Cryptococcus neoformans: involvement of IFN-gamma and its protective effect against cryptococcal infection. Microbiol. Immunol. 39, 135-143. doi: 10.1111/j.13480421.1995.tb02180.x

Kawakami, K., Tohyama, M., Teruya, K., Kudeken, N., Xie, Q., and Saito, A. (1996). Contribution of interferon-gamma in protecting mice during pulmonary and disseminated infection with Cryptococcus neoformans. FEMS Immunol. Med. Microbiol. 13, 123-130. doi: 10.1016/0928-8244(95)0 0093-3

Kelley, V. A., and Schorey, J. S. (2003). Mycobacterium's arrest of phagosome maturation in macrophages requires Rab5 activity and accessibility to iron. Mol. Biol. Cell 14, 3366-3377. doi: 10.1091/mbc.E02-12-0780

Kobayashi, M., Ito, M., Sano, K., and Koyama, M. (2001). Granulomatous and cytokine responses to pulmonary Cryptococcus neoformans in two strains of rats. Mycopathologia 151, 121-130. doi: 10.1023/A:1017900604050

Levitz, S. M., Harrison, T. S., Tabuni, A., and Liu, X. (1997). Chloroquine induces human mononuclear phagocytes to inhibit and kill Cryptococcus neoformans by a mechanism independent of iron deprivation. J. Clin. Invest. 100, 1640-1646. doi: 10.1172/JCI119688

Levitz, S. M., Nong, S. H., Seetoo, K. F., Harrison, T. S., Speizer, R. A., and Simons, E. R. (1999). Cryptococcus neoformans resides in an acidic phagolysosome of human macrophages. Infect. Immun. 67, 885-890.

Lutz, J. E., Clemons, K. V., and Stevens, D. A. (2000). Enhancement of antifungal chemotherapy by interferon-gamma in experimental systemic cryptococcosis. J. Antimicrob. Chemother. 46, 437-442. doi: 10.1093/jac/46.3.437

Ma, H., Croudace, J. E., Lammas, D. A., and May, R. C. (2006). Expulsion of live pathogenic yeast by macrophages. Curr. Biol. 16, 2156-2160. doi: 10.1016/j.cub.2006.09.032

Ma, H., Croudace, J. E., Lammas, D. A., and May, R. C. (2007). Direct cell-to-cell spread of a pathogenic yeast. BMC Immunol. 8:15. doi: 10.1186/1471-2172-8-15

Macura, N., Zhang, T., and Casadevall, A. (2007). Dependence of macrophage phagocytic efficacy on antibody concentration. Infect. Immun. 75, 1904-1915. doi: 10.1128/IAI.01258-06

Mazzolla, R., Barluzzi, R., Brozzetti, A., Boelaert, J. R., Luna, T., Saleppico, S., et al. (1997). Enhanced resistance to Cryptococcus neoformans infection induced by chloroquine in a murine model of meningoencephalitis. Antimicrob. Agents Chemother. 41, 802-807.

Mody, C. H., Tyler, C. L., Sitrin, R. G., Jackson, C., and Toews, G. B. (1991). Interferon-gamma activates rat alveolar macrophages for anticryptococcal activity. Am. J. Respir. Cell Mol. Biol. 5, 19-26. doi: 10.1165/ajrcmb/ 5.1.19

Mosberg, W. H. Jr., and Mc, A. J. (1951). Torulosis of the central nervous system; biochemical behavior of the causative organism. Bull. Sch. Med. Univ. Md. 36, $122-125$.
Nessa, K., Gross, N. T., Jarstrand, C., Johansson, A., and Camner, P. (1997). In vivo interaction between alveolar macrophages and Cryptococcus neoformans. Mycopathologia 139, 1-7. doi: 10.1023/A:10068432 02124

Nicola, A. M., Albuquerque, P., Martinez, L. R., Dal-Rosso, R. A., Saylor, C., De Jesus, M., et al. (2012). Macrophage autophagy in immunity to Cryptococcus neoformans and Candida albicans. Infect. Immun. 80, 3065-3076. doi: 10.1128/IAI.00358-12

Nicola, A. M., Robertson, E. J., Albuquerque, P., Derengowski Lda, S., and Casadevall, A. (2011). Nonlytic exocytosis of Cryptococcus neoformans from macrophages occurs in vivo and is influenced by phagosomal $\mathrm{pH}$. MBio 2:e00167-11. doi: 10.1128/mBio.00167-11

Odom, A., Muir, S., Lim, E., Toffaletti, D. L., Perfect, J., and Heitman, J. (1997). Calcineurin is required for virulence of Cryptococcus neoformans. EMBO J. 16, 2576-2589. doi: 10.1093/emboj/16.10.2576

Okagaki, L. H., and Nielsen, K. (2012). Titan cells confer protection from phagocytosis in Cryptococcus neoformans infections. Eukaryot. Cell 11, 820826. doi: 10.1128/EC.00121-12

Okagaki, L. H., Strain, A. K., Nielsen, J. N., Charlier, C., Baltes, N. J., Chretien, F., et al. (2010). Cryptococcal cell morphology affects host cell interactions and pathogenicity. PLoS Pathog. 6:e1000953. doi: 10.1371/journal.ppat.10 00953

O’Meara, T. R., Xu, W., Selvig, K. M., O’Meara, M. J., Mitchell, A. P., and Alspaugh, J. A. (2014). The Cryptococcus neoformans Rim101 transcription factor directly regulates genes required for adaptation to the host. Mol. Cell. Biol. 34, 673-684. doi: 10.1128/MCB.01359-13

Orsi, C. F., Colombari, B., Ardizzoni, A., Peppoloni, S., Neglia, R., Posteraro, B., et al. (2009). The ABC transporter-encoding gene AFR1 affects the resistance of Cryptococcus neoformans to microglia-mediated antifungal activity by delaying phagosomal maturation. FEMS Yeast Res. 9, 301-310. doi: 10.1111/j.15671364.2008.00470.x

Ost, K. S., O’Meara, T. R., Huda, N., Esher, S. K., and Alspaugh, J. A. (2015). The Cryptococcus neoformans alkaline response pathway: identification of a novel rim pathway activator. PLoS Genet. 11:e1005159. doi: 10.1371/journal.pgen.1005159

Qin, Q. M., Luo, J., Lin, X., Pei, J., Li, L., Ficht, T. A., et al. (2011). Functional analysis of host factors that mediate the intracellular lifestyle of Cryptococcus neoformans. PLoS Pathog. 7:e1002078. doi: 10.1371/journal.ppat.10 02078

Randhawa, H. S., Kowshik, T., Chowdhary, A., Preeti Sinha, K., Khan, Z. U., Sun, S., et al. (2008). The expanding host tree species spectrum of Cryptococcus gattii and Cryptococcus neoformans and their isolations from surrounding soil in India. Med. Mycol. 46, 823-833. doi: 10.1080/13693780802124026

Rittershaus, P. C., Kechichian, T. B., Allegood, J. C., Merrill, A. H. Jr., Hennig, M., Luberto, C., et al. (2006). Glucosylceramide synthase is an essential regulator of pathogenicity of Cryptococcus neoformans. J. Clin. Invest. 116, 1651-1659. doi: 10.1172/JCI27890

Saha, D. C., Goldman, D. L., Shao, X., Casadevall, A., Husain, S., Limaye, A. P., et al. (2007). Serologic evidence for reactivation of cryptococcosis in solid-organ transplant recipients. Clin. Vaccine Immunol. 14, 1550-1554. doi: 10.1128/CVI.00242-07

Salkowski, C. A., and Balish, E. (1991). A monoclonal antibody to gamma interferon blocks augmentation of natural killer cell activity induced during systemic cryptococcosis. Infect. Immun. 59, 486-493.

Shao, X., Mednick, A., Alvarez, M., van Rooijen, N., Casadevall, A., and Goldman, D. L. (2005). An innate immune system cell is a major determinant of species-related susceptibility differences to fungal pneumonia. J. Immunol. 175, 3244-3251. doi: 10.4049/jimmunol.175.5.3244

Shibuya, K., Hirata, A., Omuta, J., Sugamata, M., Katori, S., Saito, N., et al. (2005). Granuloma and cryptococcosis. J. Infect. Chemother. 11, 115-122. doi: 10.1007/s10156-005-0387-x

Smith, L. M., Dixon, E. F., and May, R. C. (2015). The fungal pathogen Cryptococcus neoformans manipulates macrophage phagosome maturation. Cell Microbiol. 17, 702-713. doi: $10.1111 / \mathrm{cmi} .12394$

Steenbergen, J. N., Shuman, H. A., and Casadevall, A. (2001). Cryptococcus neoformans interactions with amoebae suggest an explanation for its virulence and intracellular pathogenic strategy in macrophages. Proc. Natl. Acad. Sci. U.S.A. 98, 15245-15250. doi: 10.1073/pnas.261418798 
Stephen, C., Lester, S., Black, W., Fyfe, M., and Raverty, S. (2002). Multispecies outbreak of cryptococcosis on southern Vancouver Island, British Columbia. Can. Vet. J. 43, 792-794.

Stukes, S. A., Cohen, H. W., and Casadevall, A. (2014). Temporal kinetics and quantitative analysis of Cryptococcus neoformans nonlytic exocytosis. Infect. Immun. 82, 2059-2067. doi: 10.1128/IAI.01503-14

Tenor, J. L., Oehlers, S. H., Yang, J. L., Tobin, D. M., and Perfect, J. R. (2015). Live imaging of host-parasite interactions in a zebrafish infection model reveals cryptococcal determinants of virulence and central nervous system invasion. MBio 6, e1425-15. doi: 10.1128/mBio.01425-15

Thinyane, K. H., Motsemme, K. M., and Cooper, V. J. (2015). Clinical presentation, aetiology, and outcomes of meningitis in a setting of high HIV and TB Prevalence. J. Trop. Med. 2015:423161. doi: 10.1155/2015/423161

Trevijano-Contador, N., Herrero-Fernandez, I., Garcia-Barbazan, I., Scorzoni, L., Rueda, C., Rossi, S. A., et al. (2015). Cryptococcus neoformans induces antimicrobial responses and behaves as a facultative intracellular pathogen in the non mammalian model Galleria mellonella. Virulence 6, 66-74. doi: $10.4161 / 21505594.2014 .986412$

Tucker, S. C., and Casadevall, A. (2002). Replication of Cryptococcus neoformans in macrophages is accompanied by phagosomal permeabilization and accumulation of vesicles containing polysaccharide in the cytoplasm. Proc. Natl. Acad. Sci. U.S.A. 99, 3165-3170. doi: 10.1073/pnas.0527 02799

Villamil Giraldo, A. M., Appelqvist, H., Ederth, T., and Ollinger, K. (2014). Lysosomotropic agents: impact on lysosomal membrane permeabilization and cell death. Biochem. Soc. Trans. 42, 1460-1464. doi: 10.1042/BST20140145

Voelz, K., and May, R. C. (2010). Cryptococcal interactions with the host immune system. Eukaryot. Cell 9, 835-846. doi: 10.1128/EC.00 039-10

Weber, S. M., Levitz, S. M., and Harrison, T. S. (2000). Chloroquine and the fungal phagosome. Curr. Opin. Microbiol. 3, 349-353. doi: 10.1016/S13695274(00)00102-8

Wozniak, K. L., and Levitz, S. M. (2008). Cryptococcus neoformans enters the endolysosomal pathway of dendritic cells and is killed by lysosomal components. Infect. Immun. 76, 4764-4771. doi: 10.1128/IAI.00660-08
Yamaoka, H., Sakaguchi, N., Sano, K., and Ito, M. (1996). Intravascular granuloma induced by intravenous inoculation of Cryptococcus neoformans. Mycopathologia 133, 149-158. doi: 10.1007/BF02373022

Young, B. J., and Kozel, T. R. (1993). Effects of strain variation, serotype, and structural modification on kinetics for activation and binding of C3 to Cryptococcus neoformans. Infect. Immun. 61, 2966-2972.

Zaragoza, O., Alvarez, M., Telzak, A., Rivera, J., and Casadevall, A. (2007). The relative susceptibility of mouse strains to pulmonary Cryptococcus neoformans infection is associated with pleiotropic differences in the immune response. Infect. Immun. 75, 2729-2739. doi: 10.1128/IAI.00094-07

Zaragoza, O., Chrisman, C. J., Castelli, M. V., Frases, S., Cuenca-Estrella, M., Rodriguez-Tudela, J. L., et al. (2008). Capsule enlargement in Cryptococcus neoformans confers resistance to oxidative stress suggesting a mechanism for intracellular survival. Cell. Microbiol. 10, 2043-2057. doi: 10.1111/j.14625822.2008.01186.x

Zaragoza, O., Garcia-Rodas, R., Nosanchuk, J. D., Cuenca-Estrella, M., Rodriguez-Tudela, J. L., and Casadevall, A. (2010). Fungal cell gigantism during mammalian infection. PLoS Pathog. 6:e1000945. doi: 10.1371/journal.ppat.1000945

Zaragoza, O., Taborda, C. P., and Casadevall, A. (2003). The efficacy of complement-mediated phagocytosis of Cryptococcus neoformans is dependent on the location of $\mathrm{C} 3$ in the polysaccharide capsule and involves both direct and indirect C3-mediated interactions. Eur. J. Immunol. 33, 1957-1967. doi: 10.1002/eji.200323848

Conflict of Interest Statement: The authors declare that the research was conducted in the absence of any commercial or financial relationships that could be construed as a potential conflict of interest.

Copyright (c) 2016 DeLeon-Rodriguez and Casadevall. This is an open-access article distributed under the terms of the Creative Commons Attribution License (CC BY). The use, distribution or reproduction in other forums is permitted, provided the original author(s) or licensor are credited and that the original publication in this journal is cited, in accordance with accepted academic practice. No use, distribution or reproduction is permitted which does not comply with these terms. 